\title{
Mindless reading: Eye-movement characteristics are similar in scanning letter strings and reading texts
}

\author{
FRANÇOISE VITU and J. KEVIN O'REGAN \\ Université René Descartes, Paris, France \\ and \\ ALBRECHT W. INHOFF and RICHARD TOPOLSKI \\ State University of New York, Binghamton, New York
}

\begin{abstract}
The purpose of the present study was to compare the oculomotor behavior of readers scanning meaningful and meaningless materials. Four conditions were used-a normal-text-reading control condition, and three experimental conditions in which the amount of linguistic processing was reduced, either by presenting the subjects with repeated letter strings or by asking the subjects to search for a target letter in texts or letter strings. The results show that global eye-movement characteristics (such as saccade size and fixation duration), as well as local characteristics (such as word-skipping rate, landing site, refixation probability, and refixation position), are very similar in the four conditions. The finding that the eyes are capable of generating an autonomous oculomotor scanning strategy in the absence of any linguistic information to process argues in favor of the idea that such predetermined oculomotor strategies might be an important determinant of eye movements in reading.
\end{abstract}

During one century of research on eye movements in reading, several divergent theories have been proposed to account for the variability of saccade sizes and fixation durations classically observed when people read a text. From these theories, there have emerged two main hypotheses: an oculomotor hypothesis and a processing hypothesis.

The oculomotor hypothesis was originally proposed at the beginning of the century by researchers who claimed that the main component of eye-movement guidance in reading is a preprogrammed oculomotor scanning strategy. According to this hypothesis, the eyes move forward in a rhythmic fashion by making saccades of constant length and fixations of constant duration; the known variability in saccade sizes and fixation durations results primarily from noise in the oculomotor system, and secondarily from gradual adjustments of the parameters of the rhythmic strategy to ongoing processing demands (Bouma \& de Voogd, 1974; Buswell, 1920; Dearborn, 1906; Haber, 1976; Huey, 1908; Kolers, 1976; Rayner \& McConkie, 1976; Shebilske, 1975).

More recently, in a related theory, O'Regan (1990, 1992; see also O'Regan \& Lévy-Schoen, 1987) proposed

This research was conducted while F.V. had a postdoctoral position at SUNY-Binghamton. She was supported by a Lavoisier grant from the French Ministry of Foreign Affairs. This research was also supported by Grant MH5038701 and by the Center for Cognitive and Psycholinguistic Studies at SUNY-Binghamton. We would like to thank Michael Reddix and two anonymous reviewers for helpful comments. Correspondence should be addressed to F. Vitu, Laboratoire de Psychologie Expérimentale, 28 rue Serpente, 75006 Paris, France (e-mail: vitu@idf.ext.jussieu.FR) the hypothesis that the eyes do not move with saccades of constant size, independently of the visual configuration of the text, but, rather, that they are guided by a predetermined word-based scanning mode. That is, according to this theory, while a global strategy still determines which word to fixate next and which position to aim for in a word, local tactics, based on the eyes' initial landing position in each fixated word, determine whether the word should be fixated with one or several fixations. Although the strategy and tactics are predetermined, they are adjusted locally on the basis of the lengths of the successive words, and their execution depends on low-level visuomotor constraints. Furthermore, on some occasions, the ongoing linguistic processing of the encountered words intervenes to modify the oculomotor behavior.

By contrast, according to the processing hypothesis, eye movements are determined step by step by the ongoing processing of the linguistic material encountered at each fixation. Expressed in different terms, this hypothesis has actually been the most successful during the last 20 years. The interest in such a hypothesis probably stemmed from the desire to use eye movements as online indicators of the linguistic processing occurring during reading. For example, McConkie (1979) hypothesized that saccade sizes and fixation durations are globally determined by the amount of visual material that can be extracted at each fixation (or perceptual span). On the other hand, Just and Carpenter $(1980,1987)$ proposed that eye movements are determined locally by the efficiency with which the fixated words are processed. More recently, a theory based on both McConkie's hypothesis and that of 
Just and Carpenter was proposed by Morrison (1984; see also Henderson \& Ferreira, 1990; Rayner \& Pollatsek, 1989). This theory postulates that eye movements from one word to the next are guided by shifts of attention that result from termination of processing of the attended word.

Although there have been only a few attempts to precisely test the theories cited above, the available empirical data suggest that neither the oculomotor nor the processing hypothesis is sufficient in itself to account for the oculomotor behavior observed during reading, some data favoring one hypothesis, and some lending support to the other. Those data that favor the processing hypothesis show that certain oculomotor variables depend on the efficiency of ongoing processing. For example, the gaze duration (i.e., the time the eyes spend on a word), the probability of refixating a word (i.e., the probability of making an additional fixation in the word after the initial fixation), and the duration of the individual fixations can sometimes be influenced by either the linguistic characteristics of the word (e.g., its frequency of occurrence in the language and its predictability from the linguistic context [Balota, Pollatsek, \& Rayner, 1985; Ehrlich \& Rayner, 1981; Inhoff, 1984; Inhoff \& Rayner, 1986; Pollatsek, Rayner, \& Balota, 1986; Pynte, Kennedy, \& Murray, 1991; Rayner \& Duffy, 1986; Vitu, 1991b; Zola, 1984]) or the syntactic structure of the sentence (Carpenter \& Just, 1983; Frazier \& Rayner 1982); for a review of such linguistic influences, see Rayner and Pollatsek $(1987,1989)$.

On the other hand, those data that favor the oculomotor hypothesis show that certain eye-movement measures are highly sensitive to low-level visual and oculomotor factors, rather than to linguistic factors. For example, the position at which the eyes land in an upcoming word depends not on the linguistic environment (O'Regan, 1990; Rayner \& Morris, 1992), nor the availability of the word in parafoveal vision (Inhoff, 1989b; Vitu, 1991a), but, rather, on factors such as the length of the word and the lengths of the surrounding words (O'Regan, 1979; Rayner, 1979; Vitu, 1991a), or the position, relative to the word, from which the eyes have come (the 'launch site'; Briihl \& Inhoff, 1995; McConkie, Kerr, Reddix, \& Zola, 1988). Likewise, the within-word eye behavior, although partly sensitive to the word's linguistic characteristics, can also be affected by low-level oculomotor factors: Both the probability of refixating a word and the duration of the individual fixations depend on the oculomotor activity preceding the fixation on the word (Vitu, 1993).

It remains unclear whether, as proposed in the oculomotor hypothesis, in addition to being determined by visuomotor constraints, eye movements are also dependent on predetermined oculomotor strategies. Data certainly exist that are compatible with the idea that an oculomotor rhythm drives the eyes during reading, since it has been shown that it is possible to read without the eyes actively controlling the parts of text that are fixated. For example, Bouma and de Voogd (1974) presented "linestepped" text, which shifted across the screen as though saccades were being made. They found that when the text moved with a speed similar to normal reading speed, peo- ple were able to read normally. Juola, Ward, and McNamara (1982), using an RSVP technique, showed that people could read just as well when they didn't make saccades as they did when they made them. However, as pointed out by Hochberg (1975), although these experiments show that reading is possible when the eyes are passively presented with digestible packets of words, they do not show that the eyes are actually capable of generating the autonomous scanning strategy that would provide such packets. It might be that the eyes can only move forward in the way they do in reading if the need to acquire more visual information is pulling them forward.

On the other hand, the hypothesis proposed by O'Regan $(1990,1992)$-according to which, predetermined word-based scanning strategies drive the eyes during reading - has never been directly tested. Results from reading experiments show the existence of an optimal viewing position effect - whereby the probability of refixating words is smaller the closer the eyes land to the center of words (McConkie, Kerr, Reddix, Zola, \& Jacobs, 1989; O'Regan \& Lévy-Schoen, 1987; Vitu, O'Regan, \& Mittau, 1990), and these results are certainly compatible with the existence of the kind of local tactics suggested by O'Regan $(1990,1992)$. It would only be when the eyes land far from the center of a word that a within-word refixation would be automatically programmed. However, recent data obtained by Nazir (1991) in a situation where subjects were presented with isolated meaningless letter strings which did not need to be scanned for processing do not give strong support to this hypothesis. Although the refixation probability was found to depend strongly on the eyes' initial fixation position, as would be predicted by the strategy-tactics theory, there were some differences between the pattern of refixations within the words and that within the letter strings. While the probability of refixating a word increased when the eyes landed either near the word's beginning or near its end, the probability of refixating a letter string increased only when the eyes were located near its beginning. This difference suggests that within-word refixations are not caused by predetermined oculomotor tactics, but, instead, that they result, at least partly, from oculomotor aiming errors; that is, the saccade programmed to leave a letter string or a word from its beginning would actually give rise to an additional fixation on the letter string or word. However, since Nazir's data were obtained with letter strings that were presented in isolation, further experiments are necessary to test the hypothesis proposed by O'Regan $(1990,1992)$.

It is thus clear that the variability of the oculomotor behavior commonly observed during reading depends both on the necessities of ongoing processing and on visuomotor constraints. However, two further possible hypotheses exist to account for this variability, both of which depend on the role of predetermined oculomotor strategies. The first hypothesis assumes that although ongoing linguistic processing is indeed the driving force behind the eye, the strength of visuomotor constraints is such that they mask the behavior that would be expected to result from the necessities of ongoing linguistic process- 
ing. In particular, it is known that accurate saccade programming takes time, and that the eye cannot always attain an aimed-for position (Coeffé \& O'Regan, 1987; Findlay, 1982; Vitu, 1991a; Viviani \& Swensson, 1982). Such constraints might be present during text reading.

The alternative hypothesis is that linguistic processing might in fact be too slow, and therefore might not be the driving force behind eye movements; instead, there might be an underlying oculomotor strategy, that is independent of processing, and that is the main determinant of eye movements, with linguistic processing intervening only occasionally to modify the predetermined oculomotor strategy (O'Regan, 1990, 1992). Indeed, there are some data showing that the influence of linguistic factors on fixation durations is often apparent only on the fixations that follow those during which information has been gathered (Hogaboam, 1983; Inhoff \& Rayner, 1986; McConkie, Underwood, Zola, \& Wolverton, 1985; O'Regan \& Lévy-Schoen, 1987).

In order to distinguish between these two alternatives, it is necessary first to test the role of predetermined oculomotor scanning strategies in the determination of eye movements in reading. This is difficult to do in normal reading, because it is usually possible to claim that it is actually the processing of the text material that is present that is generating what appears to be an autonomous oculomotor strategy. One way of showing the plausibility that autonomous oculomotor strategies might be acting during reading is to show that the pattern of eye movements observed in this situation can actually be generated in the absence of any linguistic information to be processed.

The idea behind the present experiment was therefore to present subjects with tasks or material that involved little or no linguistic processing (i.e., with those that were orthographic, lexical, or syntactic), and to see whether the subjects' eyes moved in a way similar to their movement during normal reading. For this purpose, we used four conditions. The first- " normal text reading"- - served as the control condition, and we compared oculomotor behavior in this condition to that in three experimental conditions in which the amount of linguistic processing was reduced.

In the $z$-text-reading condition, the material consisted of homogeneous strings of the letter $z$ that were constructed by replacing all letters of the original text with the letter $z$ (upper or lower case as appropriate). For instance, the phrase "Several nocturnal animals were observed" was changed to "Zzzzzzz zzzzzzzzz zzzzzzz zzzz zzzzzzzz." The subjects were asked to scan these $z$ letter strings as though they were reading. A possible problem with this manipulation is that whereas it undoubtedly diminishes the amount of visual and linguistic processing that needs to be done in the task, it cannot be known exactly what task the subjects set for themselves when they perform the $z$-text-reading task. To overcome this potential problem, we used two additional experimental conditions-normal-text search and $z$-text search - whereby the subjects were presented with stimuli consisting either of normal text or of letter strings constructed by replacing all letters of the original text, except the letter $c$, with the letter $z$. Their task was to note all occurrences of the letter $c$ via a manual keypress response.

In order to test the hypothesis that the eyes are capable of generating an autonomous oculomotor scanning strategy in the absence of linguistic processing, we asked (1) whether the global characteristics of eye movements, such as saccade sizes and fixation durations, changed when going from the normal-text-reading control condition to the three experimental conditions in which linguistic processing was reduced to a minimum; and (2) whether, in the three experimental conditions, the local behavior in the vicinity of each word (or letter string) (such as the probability to skip or to refixate a word or a string and the fixation positions in words or strings) resembled that observed in the normal-text-reading condition. If global and/or local eye-movement parameters in the "mindless" reading conditions were found to resemble those of normal reading, this would provide support for the possibility that oculomotor strategies can be generated even when no linguistic processing is necessary, and would make plausible the existence of such strategies in normal reading.

\section{METHOD}

\section{Subjects}

Twenty-four undergraduate psychology students at SUNY-Binghamton participated for course credit. All met the following criteria: They had uncorrected normal vision, they were native speakers of English, they rated themselves as "fluent readers," and they were naive with respect to the purpose of the experiment.

\section{Materials}

The materials used in the present experiment were also used in a previously published study (Inhoff, Topolski, Vitu, \& O'Regan, 1994).

Sixteen passages of text of equal difficulty were obtained from national news magazines that were several months old. Each passage contained $75-90$ words and had a single theme. In all 16 passages combined, there was a total of 57 single-letter words, 217 twoletter words, 341 three-letter words, 272 four-letter words, 163 five-letter words, 156 six-letter words, 146 seven-letter words, 76 eight-letter words, 57 nine-letter words, and 37 ten-letter words. Each passage contained approximately the same number of words of each length. The sequence of 16 passages was constant across the four experimental conditions, and all passages were shown in successive one-line displays. The passages will be referred to as standard text. A sample passage, as it appeared on the computer screen to the subjects, is shown in Table 1.

The standard text was converted to letter strings by replacing, in the $z$-text-reading condition, each letter of the text with the letter $z$, and in the $z$-text-search condition, every letter, except for the letter $c$, with the letter $z$. Four lists of 16 passages were devised, each containing the same sequence of passages. In List 1, Passages 1-4 and 9-12 were normal text; in List 2, Passages 1-8 were normal text; in List 3, Passages 9-16 were normal text; and in List 4, Pas- 
Table 1

\section{Sample Passage}

There is a fairly good possibility that the Buffalo Bills will win the Super Bowl this season. It seems that their biggest problem is internal quarreling. With good leadership they should be able to overcome this dilemma. As long as Jim Kelly and Thurman Thomas continue to play as they did last year, the offense will lead the league in most ever statistic. On the other side of the football, the defense needs to return to the dominating style of play it was known for two years ago. If the Bills are able to do this, they could win the Super Bowl this year.

sages 5-8 and 13-16 were normal text. All other text consisted of derived letter strings. Each subject saw a list of 16 passages (consisting of 4 different passages in each condition). The condition to which passages were assigned was counterbalanced across subjects, so that (1) each subject saw each passage only once, and (2) each passage was seen in all four conditions across subjects.

\section{Apparatus}

A $60-\mathrm{Hz}$ Dell VGA color plus monitor interfaced with an IBMAT-compatible $486 / 33-\mathrm{MHz}$ computer was used to display text, which was shown in light green on a black background. The distance between readers' eyes and the monitor was set at $45 \mathrm{~cm}$; at this viewing distance, each letter of text subtended $.33^{\circ}$ of visual angle.

Eye movements were recorded via a fifth-generation dual-Purkinje (Stanford Research Institute) eye-tracking system, which has a spatial resolution of $10^{\prime}$ of arc, and whose output is approximately linear over the vertical and horizontal range of the VGA display. Viewing was binocular, but eye movements were recorded from the right eye only. Analog input from the eye tracker was digitized via a Data Translation A-D converter housed in the computer, which recorded and stored horizontal and vertical eye position every $2 \mathrm{msec}$. This record was used to determine the different word-viewing time measures and corresponding saccade sizes. A QWERTY keyboard and a Logitech mouse were also interfaced with the computer, which detected keyboard activation during the experiment to register detection of the critical letter $c$; the mouse served as another source of external input during the calibration routine and during the subject-paced presentation of lines of text.

\section{Procedure}

The subjects were tested individually. When a subject arrived in the laboratory, a bitebar was positioned so as to reduce head movements during the experiment. The session began with a two-dimensional calibration of the eye-tracking system, during which the subject was requested to fixate four monitor positions (left top, right top, left bottom, right bottom) as they appeared sequentially on the screen. The subject was asked to fixate accurately each illuminated position and to manually depress a mouse button while fixating each position. The computer sampled the $x / y$-coordinates of a subject's fixation location during the $150-\mathrm{msec}$ following mouse activation.

After calibration was completed, six character-size boxes appeared simultaneously on the computer screen (at the top left, top right, bottom left, and bottom right, and $4 \mathrm{~cm}$ to the right and to the left of center). During this calibration-check phase, the subject's eye position was plotted on the screen so that it appeared as a yellow cursor the size of one character, that moved in synchrony with the eyes. The calibration was considered successful when this computergenerated eye position (i.e., the yellow cursor) deviated by no more than one character space from the subject's actual eye position (i.e., the illuminated green character space) in each of the six fixation locations. Recalibrations were performed when larger errors were observed.

After successful calibration, the subject was asked to fixate a marker at the left side of the screen and to depress a response but- ton on the mouse to display a line of text. The first letter of text of each line appeared approximately $2.5 \mathrm{~cm}$ to the right of the fixation marker. Another pressing of a mouse button erased the display and triggered the presentation of the left-side fixation marker Similar mouse activations preceded and followed the presentation of all lines of text and letter strings. In the $c$-detection condition, the subject was asked to press the space bar on the keyboard each time a target letter was detected.

In both text conditions, reading for meaning was encouraged. After each passage, the subject was asked to come off the bitebar and to provide a short summary of the passage (in both reading conditions) and answer questions about the details of the passage. All of the subjects were able to report the global passage content correctly in the two text conditions and generally were also able to answer the questions correctly.

No formal examination of letter-detection accuracy in the search conditions was performed, as attainment of a particular performance level was not considered critical for the purpose of the study. However, cursory examination of detection accuracy indicated that the subjects neither detected more $c$ letters than were actually present nor missed them more than $20 \%$ of the time they actually occurred. This indicates that subjects effectively followed the task instructions.

Prior to the experiment, the subjects read four sample passages, each illustrating a different experimental condition. All of the subjects were instructed to move their eyes along derived text (i.e., strings of $z \mathrm{~s}$ ) as if they were reading, and were told that there was no hidden meaning in the $z$-text conditions.

\section{Data Selection}

As with earlier reading studies (e.g., Balota et al., 1985; Inhoff, 1989a, 1989b; Lima \& Inhoff, 1985), a target word was considered fixated when the point of fixation fell either on one of its constituent letters or on the blank space preceding it. Only those fixations that lasted $50 \mathrm{msec}$ or more were analyzed. Instances in which the specification of $x / y$-coordinates was unclear (i.e., track losses) and occasional blinks resulted in the loss of approximately $5 \%$ of the data. No fixation data were available from words that did not receive a fixation. A word was considered as having been skipped when the last fixation before it during the first reading pass was followed by a progressive saccade that brought the eyes beyond the last letter of that word. Such word skippings were counted and converted to percentage values. Skipping rates, initial landing sites in words, and refixation probabilities were analyzed using within-subjects analyses of variance (ANOVAs). The main withinsubject factor used in the analyses was scanning condition, with four levels. Other within-subject factors, such as word length, launch site, or initial fixation position in words, were defined a posteriori.

Means or proportions were calculated for each subject, for each dependent variable considered in the Results section, and these were then averaged across subjects. Thus, the weights of individual subjects' contributions to the final values were not influenced by the number of fixations that qualified for a particular cell. In the ANOVA, each individual mean or proportion was considered as an observation.

\section{RESULTS}

\section{Global Eye-Movement Characteristics}

The first finding to be noted concerning the present manipulations is that the subjects had no problem doing the task. Both when they were asked to move their eyes as though they were reading and when they were searching for a target letter, their oculomotor behavior closely resembled that during normal reading. Although there were differences between the conditions, lines were al- 
ways scanned with a regular sequence of fixations and saccades.

In Figures 1 and 2, which present, respectively, the distributions of saccade sizes and the distributions of fixation durations, it can be seen that the shape of both distributions in the normal-text-reading control condition is very similar to the shape of the corresponding distributions observed in the three experimental conditions (normaltext search, $z$-text reading, and $z$-text search). In all four conditions, the distributions are unimodal and centered around the same modal values ( 7.5 characters for saccade sizes and $225 \mathrm{msec}$ for fixation durations).

A closer examination of the obtained distributions, however, reveals differences between the conditions. First, in the normal-text-reading control condition, compared with the three experimental conditions, there are fewer very small progressive saccades (i.e., of about 2.5 characters long) and more long saccades (i.e., of about 12.5 characters long). Second, fixation durations are globally shorter in the normal-text-reading condition than they are in the three experimental conditions. Third, while the distributions of saccade sizes observed in the three experimental conditions do not differ from each other, there is a clear difference in those obtained for fixation durations, between the normal-text-search condition and both the $z$-text-reading and the $z$-text-search conditions, the distributions for both of the latter conditions being almost superposed.

To determine statistically both whether and how the distributions of saccade sizes and fixation durations differed between the normal-text-reading control condition and the three experimental conditions, two factors were defined a posteriori-namely, the class of saccade sizes (with 9 levels, from -17.5 letters up to 22.5 letters in 5-letter steps) and the class of fixation durations (with 10 levels - going from 125 up to $575 \mathrm{msec}$ in 50 -msec steps). Following that, a one-way comparison of the normal-

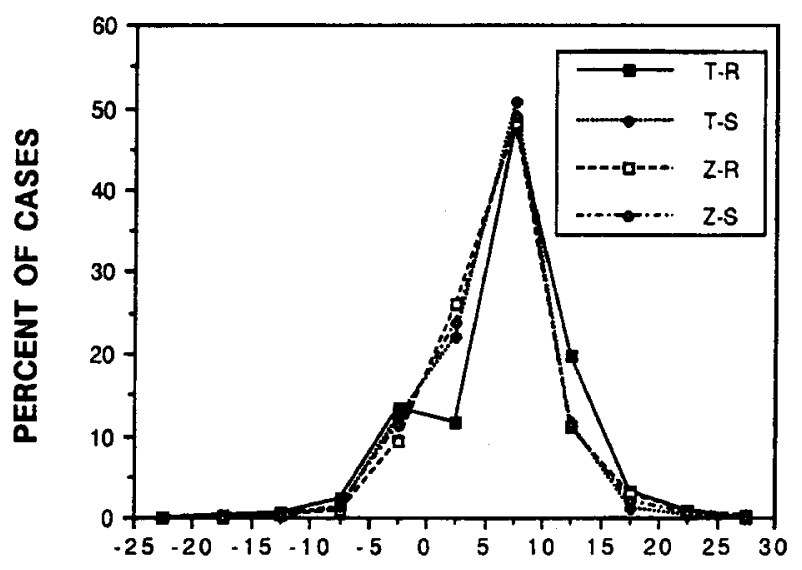

SACCADE SIZES (LETTERS)

Figure 1. Global distribution of saccade sizes (in characters) in the normal-text- and $z$-text-reading conditions (T-R and $Z-R$ ), and in the normal-text- and $z$-text-search conditions (T-S and Z-S).

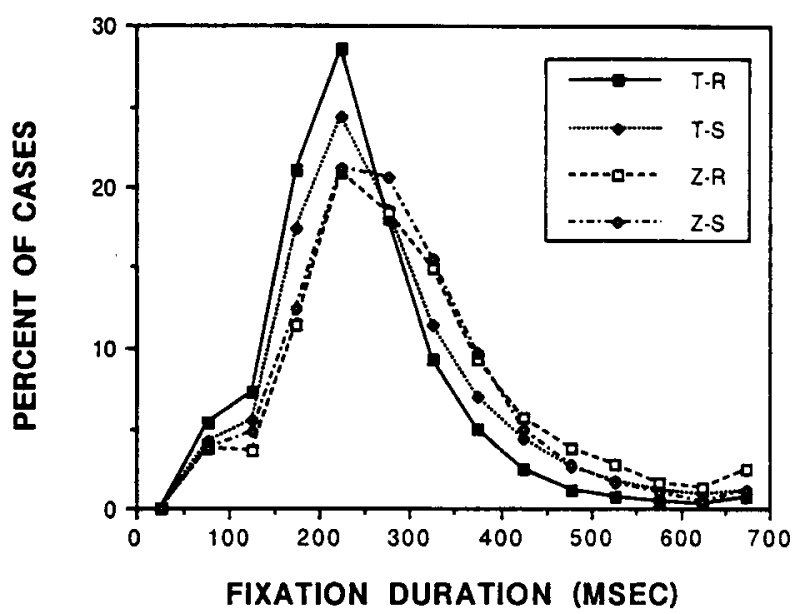

Figure 2. Global distribution of fixation durations (in msec) in the normal-text- and $z$-text-reading conditions (T-R and Z-R), and in the normal-text- and $z$-text-search conditions ( $T-S$ and $Z-S$ ).

text-reading control condition with all three experimental conditions grouped was performed for each class of both saccade sizes and fixation durations.

This analysis revealed that although there was a significant difference for saccade sizes in the range of $-12.5-2.5$ characters and for saccade sizes of 12.5 characters, there was no significant difference for the most frequently observed saccade size, of 7.5 characters $[t(23)=1.21$, n.s.; $t(23)=$ $2.51, p<.025 ; t(23)=2.49, p<.025 ; t(23)=3.19, p<$ $.005 ; t(23)=6.43, p<.0005 ; t(23)=1.12$, n.s.; $t(23)=$ $5.57, p<.0005 ; t(23)=1.73, p<.10 ; t(23)=.96$, n.s., respectively, for the 9 classes of saccades sizes]. The interaction between the scanning condition and the class of saccade sizes was significant $[F(24,552)=6.76, p<.0005]$.

On the other hand, there was a significant difference between the normal-text-reading control condition and all three experimental conditions grouped for each class of fixation duration [ $p<.05$ or less, with $t(23) \geq 2.12$ ]. The interaction between the scanning condition and the class of fixation durations was also significant $[F(27,621)$ $=13.31, p<.0005]$.

In conclusion, it appears that the patterns of eye movements adopted by subjects while reading a text, while searching for a target letter, and while moving the eyes through lines of meaningless letter strings clearly resemble each other. This shows that people are able to generate an oculomotor strategy driven by the purely visual characteristics of the page of print, regardless of its content; whether they are looking for a target letter or moving their eyes across meaningless letter strings, their behavior is almost indistinguishable from that observed during normal reading. However, as reported above, there are some differences both in the durations of fixations and, to a certain extent, in the sizes of saccades as a function of the stimulus type (text or $z$-letter strings) and the task given to the subjects (reading or searching for a target letter). 
In the next section, we will analyze in detail the oculomotor behavior adopted locally in the vicinity of words or $z$-letter strings (in terms of skipping probability, landing sites, refixation probability, and positions of refixations), in order to determine to what extent the scanning condition changes the pattern of eye movements between and within words.

\section{Local Eye-Movement Characteristics}

Skipping probability. It is known that during text reading, 35\% of the words are skipped, and also that the probability of skipping a word is a function of the length of the word, short words being skipped more often than long words (Blanchard, Pollatsek, \& Rayner, 1989; Carpenter \& Just, 1983; Just \& Carpenter, 1987; O'Regan, 1979, 1990). It is also known that the probability of skipping a word depends strongly on the position from which the eyes are launched (the launch site), words close to the fixation point being skipped more often than words farther from the fixation point (Kerr \& McConkie, 1992).

The results obtained in the normal-text-reading control condition of the present experiment are compatible with previous data, since they follow exactly the same pattern. It was found that $42 \%$ of the words were skipped, and that short words were skipped more often than long words [the effect of word length was significant in this condition: $F(9,207)=268.69, p<.0005$; see Figure 3]. Furthermore, words that were close to the fixation point (i.e., words whose initial letter was located between 2 and 4 characters away from the previous fixation position) were skipped more frequently than words that were farther from the fixation point (i.e., words whose initial letter was located more than 4 characters away from the previous fixation position). The effect of launch site was significant in this condition for all word lengths except 9 - and 10 -letter words $[p<.025$ or less, with $t(23) \geq 2.49$

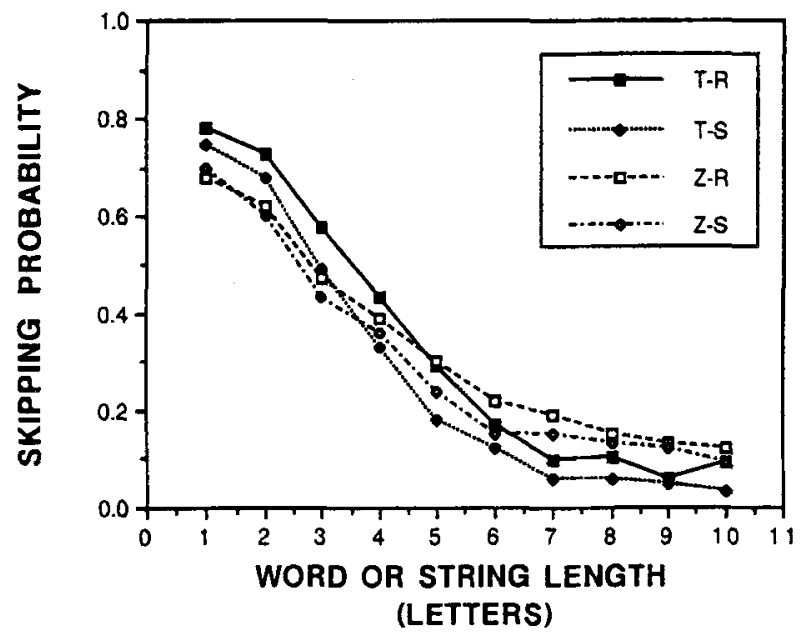

Figure 3. Word- (or letter-string-) skipping probability as a function of word (or letter-string) length in the normal-text- and $z$-textreading conditions (T-R and $Z-R$ ) and in the normal-text- and $z$-textsearch conditions (T-S and Z-S). for 1-8-letter words; $t(23)=1.81$, n.s. and $t(23)=.14$, n.s. for 9-and 10-letter words, respectively; see Figure 4a].

The results for the three experimental conditions ( $z$-text reading, normal-text search and $z$-text search) were similar to the results for the normal-text-reading control condition (see Figures 3 and $4 a-d$ ). The global effect of length of letter string or word was also significant in these three conditions $[F(9,207)=99.50, p<.0005 ; F(9,207)=$ $298.69, p<.0005$; and $F(9,207)=130.87, p<.0005$, for the $z$-text-reading, normal-text-search and $z$-text-search conditions, respectively]. For at least 1-5-letter words, the effect of launch site was also significant in the three experimental conditions $[p<.005$ or less, with $t(23) \geq$ 3.58 , for $1-5$-letter words in the $z$-text-reading condition; $p<.01$ or less, with $t(23) \geq 2.93$, for $1-5$-letter words in the normal-text-search condition; $p<.05$ or less, with $t(23) \geq 2.28$, for $1-5$-letter words as well as 7 -letter words in the $z$-text-search condition].

It is interesting to note that while for short words (or letter strings) the skipping rate is higher in the normaltext-reading control condition than it is in the three experimental conditions, for long words, it is highest in the $z$-text-reading condition (see Figure 3 ). There was a significant effect of scanning condition on word skipping $[F(3,69)=3.26, p<.025]$, as well as a significant interaction between scanning condition and word length $[F(27,621)=6.02, p<.0005]$. This was the case both for close launch sites $[F(3,69)=4.28, p<.01 ; F(27,621)=$ 2.33, $p<.0005$, for the global effect and the interaction, respectively] and for far launch sites $[F(3,69)=4.56$, $p<.005 ; F(27,621)=5.22, p<.0005$, for the global effect and the interaction, respectively]. One-way comparisons of the normal-text-reading control condition with each of the three experimental conditions for each word length showed that the normal-text-reading condition actually only differed significantly from the $z$-text-reading condition for very short words (i.e., up to 3 letters long) and long words [ 7 and 9 letters long; $p<.025$ or less, with $t(23) \geq 2.41$ for $1-, 2-, 3-, 7$-, and 9-letter words]. On the other hand, the normal-text-reading condition differed from both search conditions only for short words [i.e., those up to 4-5 letters long; normal-text reading/ normaltext search: $p<.05$ or less, with $t(23) \geq 2.12$ for $2-5$ letter words; normal-text reading/ $z$-text search: $p<.025$ or less, with $t(23) \geq 2.41$ for $1-4$-letter words]. In other words, while going from normal-text reading to $z$-text reading reduced the number of short words skipped and increased the number of long words skipped, going from normal-text reading to either normal-text search or $z$-text search only reduced the number of short words skipped.

The decreased skipping probability in both the $z$-textreading and the $z$-text-search conditions compared with the normal-text-reading control condition probably resulted from differences in the degree of usefulness of parafoveal preprocessing for the two types of materials: It is probably more useful to preprocess words than to preprocess $z$-letter strings. Since short words can be more easily preprocessed than long words in parafoveal vision, word-skipping rate is higher for text-type stimuli 
a) normal text reading
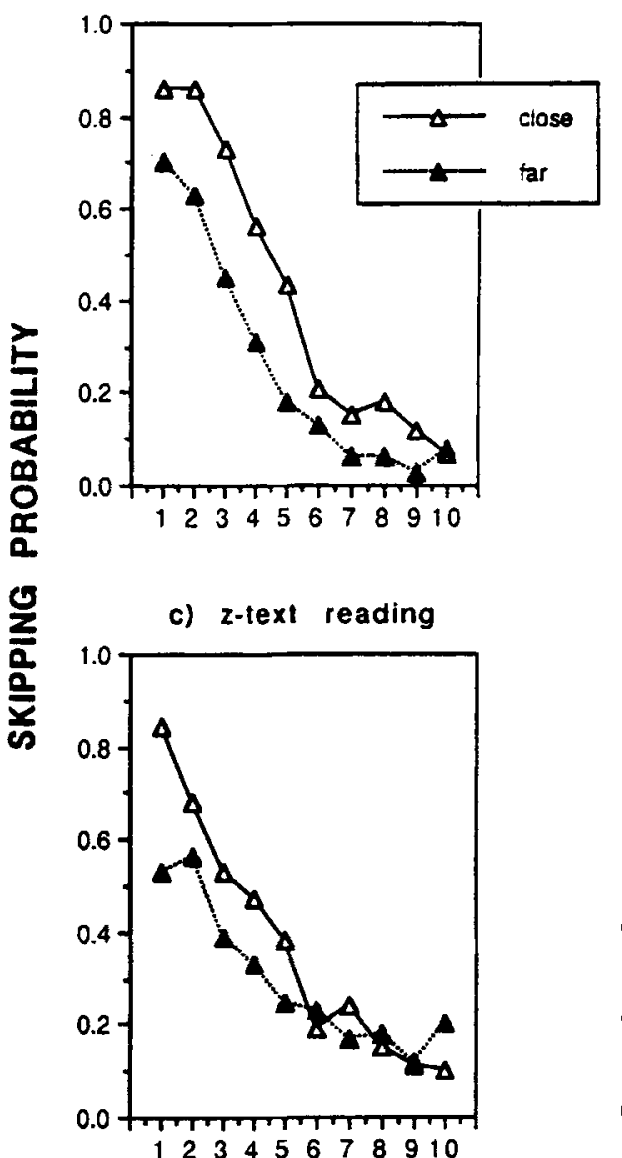

b) normal text search

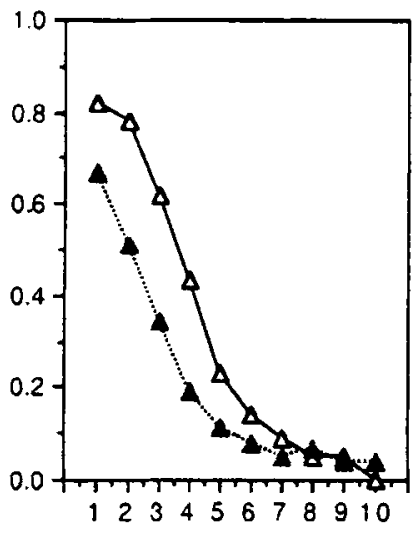

d) z-text search

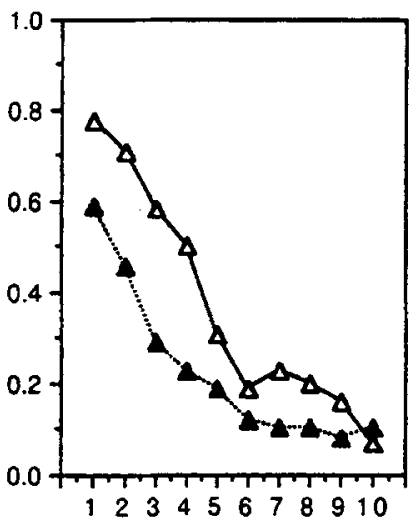

\section{WORD OR STRING LENGTH (LETTERS)}

Figure 4. Word- (or letter-string-) skipping probability as a function of word (or letterstring) length and the position, relative to the word, from which the eyes were launched (close = between 2 and 4 characters away from the initial letter of the word; far = more than 4 characters away), in the normal-text- and $z$-text-reading conditions (a and $c$ ), and in the normal-text- and $z$-text-search conditions (b and d).

than for $z$-letter-string stimuli only in the case of short words (or letter strings). Furthermore, the time spent processing a word in parafoveal vision is probably short, and may be too short for the target-letter detection process to be carried out before the programming of the next saccade is terminated. This would explain why the skipping probability is smaller in the normal-text-search condition than it is in the normal-text-reading condition.

In conclusion, although there are some differences between the normal-text-reading control condition and the three experimental conditions, it appears that the patterns most commonly observed during normal-text reading-namely, that short words and words close to the fixation point are more often skipped-are clearly present and quite similar when searching for a target letter or when scanning meaningless letter strings. The only difference between the normal-text-reading condition and the three experimental conditions lies in the usefulness during normal reading of parafoveal processing, which slightly increases the probability of skipping short words in that condition.

We will now turn to the cases in which a word or a letter string is fixated and consider several characteristics of the oculomotor behavior-namely, the initial eyelanding position in words or letter strings, the probability of refixating words or letter strings, and the positions of the second fixations in the cases where a within-word (or within-letter string) refixation occurs. The data selected for this analysis correspond to each fixated word's first reading pass preceded by a forward saccade.

Initial landing sites in words. During text reading, the eyes generally land between the beginning and the middle of words at what has been called the preferred landing position (McConkie et al., 1988; Rayner, 1979; Vitu et al., 1990). In the normal-text-reading control condition of the present experiment, the pattern of landing sites is quite similar; as can be seen in Figures $5 \mathrm{a}-\mathrm{e}$, which present the distributions of initial landing sites on 5-, 6-, 7-, 
a) 5-lotter words

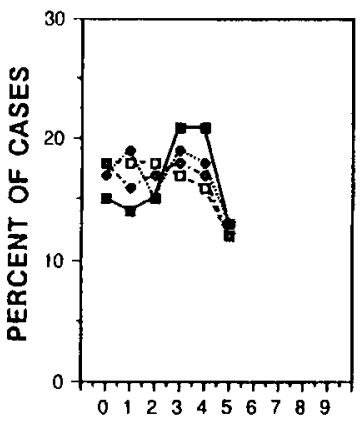

b) 6-lelter words

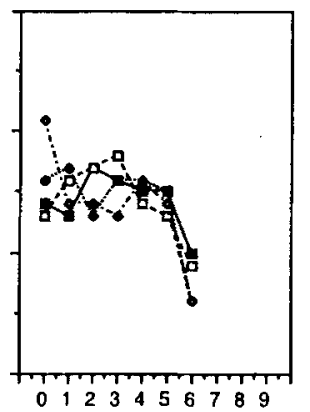

c) 7-letter words

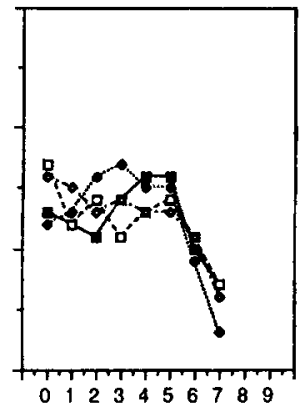

d) 8-letter words

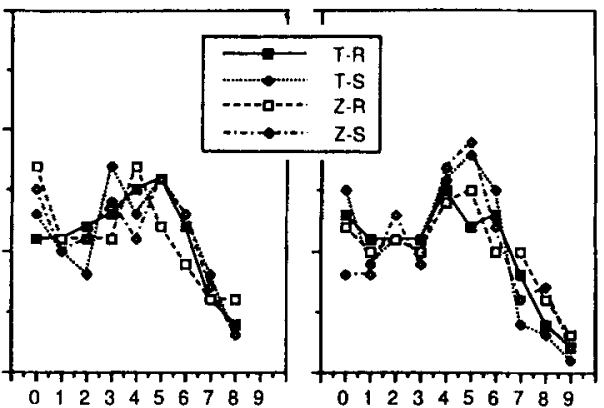

LANDING SITES (LETTERS)

Figure 5. Distribution of initial landing sites in 5-9-letter words or $z$-strings (a-e), for the normal-text- and $z$-text-reading conditions (T-R and Z-R) and the normal-text- and $z$-text-search conditions (T-S and Z-S). (On the abscissa, Letter 0 corresponds to the blank space before the word.)

8-, and 9-letter words and letter strings (as well as on the blank space before these words), the eyes mostly land near the middle of words. In the three experimental conditions, the landing-site distributions are quite similar to that obtained in the normal-text-reading condition, but primarily so in the cases of 8-and 9-letter words. For words less than 8 letters in length, there are more instances (compared with the normal-text-reading control condition) in which the eyes land toward the beginning of the words.

In order to compare the distributions obtained in the four conditions, an ANOVA was performed after defining the landing site as a within-subject factor, with 6,7 , 8, 9, and 10 levels for 5-, 6-, 7-, 8-, and 9-letter words, respectively (since the blank space before the word was included in the analysis). This analysis showed that for all words except 6- and 7-letter words, there was no significant difference between the normal-text-reading control condition and the three experimental conditions grouped for all landing sites $[t(23) \leq 1.89, p<.10$; $t(23) \leq 1.37$, n.s.; and $t(23) \leq 1.96, p<.10$, for $5-, 8$, and 9-letter words, respectively]. The difference was sig- nificant for some of the landing sites in the cases of 6 letter words $[t(23)=2.11, p<.05$, for Letter 6] and 7-letter words $[t(23)=2.11, p<.05$, for Letter 2]. The interaction between scanning condition and landing site was significant only for 6- and 7-letter words $[F(15,345)=1.09$, n.s.; $F(18,414)=2.50, p<.001 ; F(21,483)=1.81, p<.025$; $F(24,552)=.96$, n.s.; and $F(27,621)=1.04$, n.s., respectively, for 5-, 6-, 7-, 8-, and 9-letter words, respectively].

In conclusion, it appears that the position at which the eyes initially land in words or letter strings when subjects are asked to search for a particular target letter, and/or when they are presented with lines of meaningful letter strings, is not strongly different from the position at which the eyes land in words during normal-text reading.

Probability of refixating words. When a word is fixated during text reading, it is sometimes fixated with a single fixation and sometimes with several fixations. The probability of refixating a word has been shown to depend strongly on the eyes' initial fixation position, in that when the eyes initially fixate near the middle of the word, the refixation probability is much smaller than it a) 5-letter words

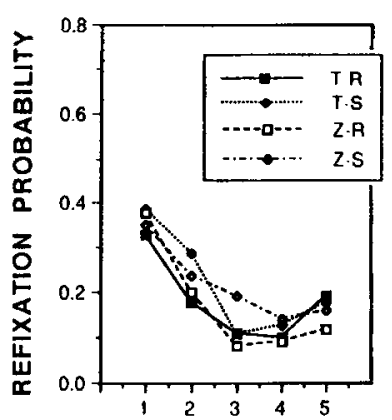

b) 6-letter words

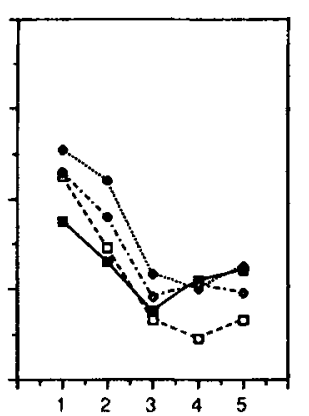

c) 7-letter words

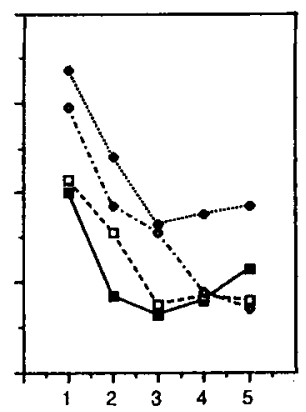

d) 8-letter words

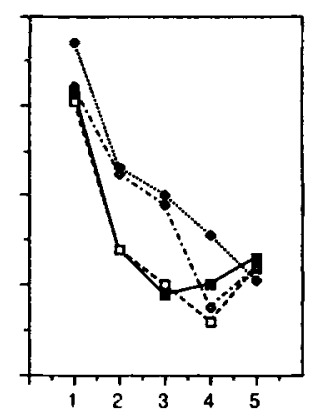

e) 9-leller words

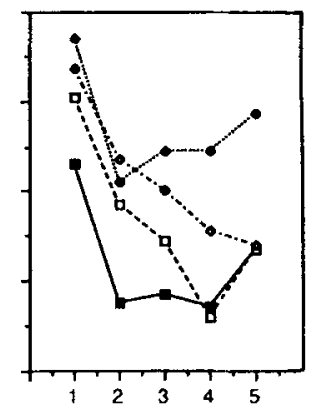

INITIAL FIXATION POSITION

(ZONES)

Figure 6. Probability of refixating words or $z$-strings of $5-9$ letters (a-e) as a function of the initial fixation position in either words (for the normal-text-reading and normal-text-search conditions [T-R and T-S]) or letter strings (for the $z$-text-reading and $z$-text-search conditions [T-S and Z-S]). See text for computation details. 
is when the eyes land toward the word's beginning or end (the optimal viewing position effect; McConkie et al., 1989; Vitu et al., 1990).

The graphs in Figures 6a-e present the probability of refixating words (or letter strings) as a function of the eyes' initial fixation position in 5-9-letter words (or letter strings), in each of the four conditions. The eyes' initial fixation position in a word (which was originally measured in letters) was computed relative to the word length using the formula $(f-.5) / w l$, where $f$ is initial fixation position and $w l$ is word length, and was then recoded in terms of five "zones" (Zones 1-5) of equal size (1/5).

As Figures 6a-e illustrate, the optimal viewing position effect in words is present not only during text reading but also (to a certain extent) in conditions where linguistic processing has been reduced to a minimum. In the four conditions, and for all word lengths, the refixation probability strongly depends on the eyes' initial fixation position; it is higher at the beginning or end of words (and particularly so at the beginning) than at the middle. The effect of the eyes' initial fixation position was significant for all word lengths in both reading conditions $[p<.05$, with $F(4,92) \geq 2.68$ in the normal-text-reading condition; and $p<.001$ or less, with $F(4,92) \geq 5.51$ in the $z$-text-reading condition], as well as in both search conditions $[p<.025$ or less, with $F(4,92) \geq 3.10$ in the normaltext-search condition; and $p<.01$ or less, with $F(4,92) \geq$ 3.73 in the $z$-text-search condition]. Furthermore, the interaction between the scanning condition and the initial fixation position was not significant for any word length $[F(12,276) \leq 1.47$ n.s. $]$.

It is interesting to note that the curves for the normaltext- and the $z$-text-reading conditions are almost superposed, as are the curves for normal-text- and $z$-text-search conditions. It thus seems that going from normal-text reading to $z$-text reading or from normal-text search to $z$-text search causes no change in the refixation probabilities. However, there does seem to be a difference between the reading task and the search task. The global effect of the scanning condition was significant for all word lengths except 5 -letter words $[F(3,69)=1.41$, n.s.; $F(3,69)=4.80, p<.01 ; F(3,69)=15.56, p<.0005$;
$F(3,69)=4.08, p<.025 ;$ and $F(3,69)=21.60, p<.0005$, respectively, for 5-, 6-, 7-, 8-, and 9-letter words]. Further analyses revealed that this global effect resulted mainly from a difference between the normal-text-reading condition and the normal-text-search condition. While the normal-text-reading condition differed significantly from the normal-text-search condition for all word lengths except 5-letter words [for which the difference was marginally significant; $p<.02$ or less, with $t(23) \geq$ 2.77 for 6-9-letter words], it differed significantly from the $z$-text-search condition only for 7-and 9-letter words [ $p<.02$ or less, with $t(23) \geq 2.80$ for 7 - and 9-letter words], and from the $z$-text-reading condition only for 9-letter words $[t(23)=2.36, p<.05]$.

Thus, although the pattern of within-word (or within letter-string) refixations as a function of the initial fixation position (or optimal viewing position effect) does not change much when going from normal-text reading to $z$-text reading, normal-text search, or $z$-text search, the global proportion of refixations does change as a function of the scanning condition. The refixation probability is globally higher in the three experimental conditions than in the normal-text-reading control condition; however, while this is true for all word lengths in the normal-textsearch condition, this effect is only present for long words in the $z$-text-reading and $z$-text-search conditions. This global increase of the refixation probability is consistent with the observation made above that a larger proportion of very small saccades occurs when scanning $z$-letter strings or searching for a target letter than when reading normal texts.

Positions of refixations in words. The positions of refixations in words are known to be systematically determined by the eyes' initial fixation position in the words: the eyes go to the other end of the word from which they started (O'Regan \& Lévy-Schoen, 1987; Vitu, 1990, 1993).

Figures $7 \mathrm{a}-\mathrm{g}$ and $8 \mathrm{a}-\mathrm{g}$ show, for the normal-text-reading condition and the $z$-text-reading condition, respectively, the distributions of the positions of the refixations in 6letter words, as a function of the eyes' initial fixation positions. The distributions obtained for other word lengths and for the normal-text-and the $z$-text-search conditions

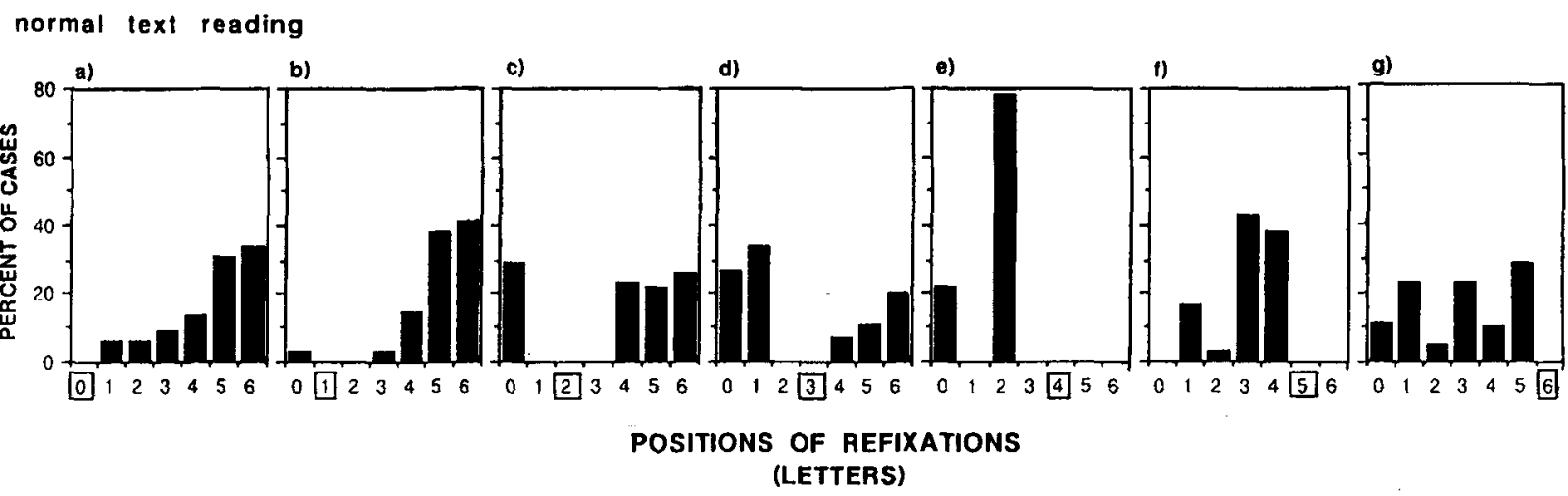

Figure 7. Distributions of the refixation positions in 6-letter words (in the 2-fixation cases) as a function of the initial letter fixated in the word (letter position enclosed in box), for the normal-text-reading condition. (On the abscissa, Letter 0 corresponds to the blank space before the word.) 


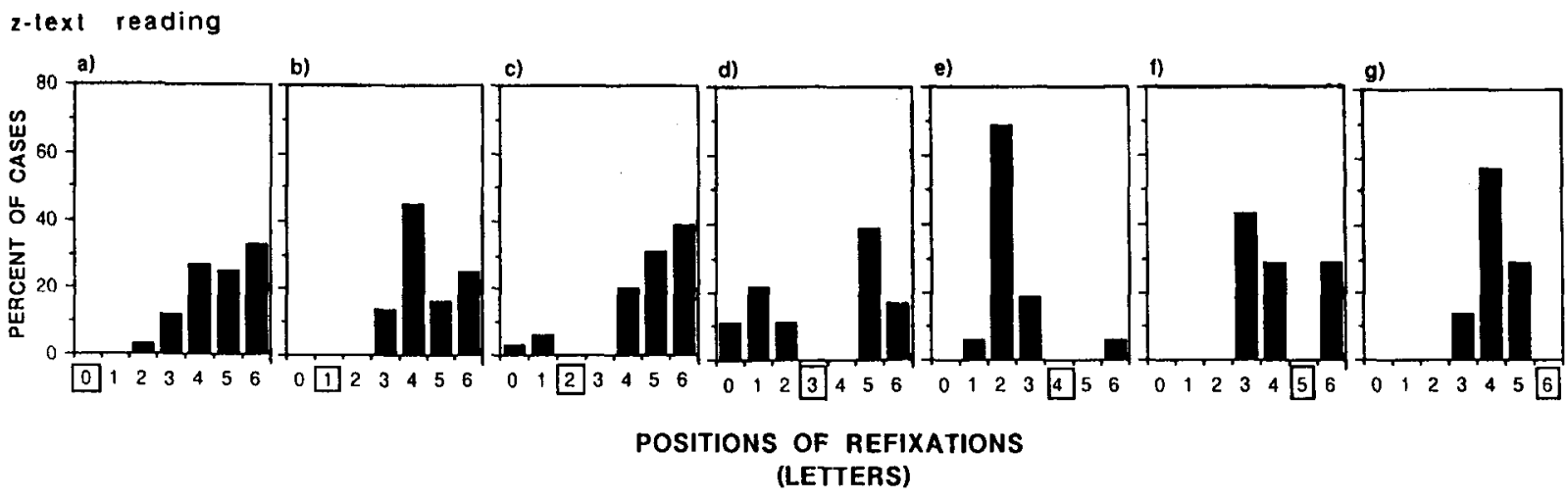

Figure 8. Distributions of the refixation positions in 6-letter strings (in the 2-fixation cases) as a function of the initial letter fixated in the letter string (letter position enclosed in box), for the $z$-text-reading condition. (On the abscissa, Letter 0 corresponds to the blank space before the word.)

were very similar. As was previously observed, the eyes tend to go to the opposite end of the word from their initial location. There is, however, a small difference between the normal-text-reading control condition and the $z$-text-reading condition, in that the eyes tend to refixate slightly closer to the ends of words than they do to the ends of $z$-letter strings. The same is true for other word (or letter-string) lengths, as well as in both search conditions in comparison with the normal-text-reading condition. Because of a lack of data, however, no ANOVA was performed for this particular analysis.

\section{DISCUSSION}

As reported in the Introduction, eye movements in reading depend not only on the visual and linguistic characteristics of the encountered words, but also on low-level visuomotor factors. A pure moment-to-moment ongoingprocessing hypothesis cannot therefore account for all the variability of the oculomotor behavior observed during reading. Instead, one of two assumptions has to be made-namely, either (1) that ongoing processing of the encountered words is the main determinant of eye movements, but that it is modulated by visuomotor constraints, or (2) that predetermined oculomotor scanning strategies are the main driving force of the eyes, and that ongoing processing intervenes only occasionally to modify the oculomotor behavior.

Since the possibility that predetermined oculomotor scanning strategies might be acting during normal text reading has never been tested, it is not possible at present to distinguish between the above two alternatives. In order to provide new data concerning this question, the present study tested whether conditions exist in which the eyes can generate oculomotor patterns similar to those observed during normal-text reading, without the need for linguistic (lexical, syntactic, and semantic) processing. If this were found to be the case, it would make it feasible for predetermined oculomotor scanning strategies to be used in normal reading.
The results obtained in the present experiment support the hypothesis that predetermined oculomotor scanning strategies are active during reading. They show that ocular behavior closely resembles the behavior observed during normal reading not only in situations in which there is a reduction in the amount of linguistic processing required (e.g., as when subjects searched for the letter $c$ in normal texts), but also in situations requiring virtually no processing (e.g., as when subjects searched for a target letter in meaningless $z$-letter strings or scanned repeated letter strings with no targets to search for).

First, during $z$-text reading, as well as during normaltext and $z$-text search, the global characteristics of eye movements (e.g., the size of saccades and, to a certain extent, the durations of fixations) were similar to those observed during normal-text reading. Second, the local characteristics of eye movements in the three experimental conditions were similar to those observed in the normaltext reading control condition, in that (1) word- or letterstring-skipping probability was dependent in the same way on the length of the word or the letter string, as well as on the position, relative to the word or letter string, from which the eyes are launched; (2) the eyes' initial landing position in words or letter strings was mainly located between the beginning and the middle of the words (or letter strings); (3) the probability of refixating words or letter strings was affected by the eyes' initial fixation position in the words or the strings, giving rise to the classical optimal viewing position effect; and (4) the positions of the refixations in words or letter strings were strongly affected by where the eyes started in the words or letter strings.

It thus appears that the eyes are capable of adopting oculomotor scanning strategies similar to those they adopt during text reading, even when there is no linguistic information to be processed. The astonishing resemblance observed between the global and local characteristics of eye movements, during normal-text reading and during the scanning of meaningless letter strings, suggests that predetermined oculomotor strategies might be an im- 
portant element in determining oculomotor behavior during normal-text reading, influencing which word to go to next, where to land in a word, and how many fixations to make on any particular word.

It is important to note, however, that despite these remarkable similarities, some differences were observed between the normal-text-reading control condition and the three experimental conditions. First, in the normaltext-reading condition, the proportion of very small saccades (of about 2.5 characters) was smaller, and the proportion of large saccades (of more than 10 characters in length) was greater than in the three experimental conditions. Second, although the distributions of fixation durations were centered around $225 \mathrm{msec}$ in all four conditions, the fixation durations were globally shorter in the normal-text-reading condition than in the three experimental conditions.

Further analyses of the oculomotor behavior adopted locally in the vicinity of each word showed that the reduction of the proportion of very small saccades in the normaltext-reading condition resulted from differences in the probability of refixating long words (or letter strings); that is, the probability of making an additional fixation on a word after the initial fixation was smaller in the text-reading condition. This might be due to the fact that linguistic constraints peculiar to text reading facilitate the identification of words (particularly long words), and therefore reduce the refixation probability in text reading. Indeed, it has been shown that the probability of refixating a word while reading is strongly affected by linguistic constraints, such as the word's frequency of occurrence in the language or its predictability from the linguistic context (Balota et al., 1985; Pynte et al., 1991; Vitu, 1991b). Likewise, the observation that fixation durations are globally shorter in the normal-text-reading condition than in the three experimental conditions could result from the same influence (Balota et al., 1985; Ehrlich \& Rayner, 1981; Inhoff, 1984; Inhoff \& Rayner, 1986; Pollatsek et al., 1986; Pynte et al., 1991; Rayner \& Duffy, 1986; Vitu, 1991b; Zola, 1984).

Thus, although predetermined oculomotor scanning strategies might participate in the determination of the within-word eye behavior during reading (as shown above by the presence of an optimal viewing position effect in both words and letter strings), factors related to the ongoing processing of the fixated word also influence the within-word eye behavior. To account for this double influence, we could assume that the within-word eye behavior is mainly driven by oculomotor tactics based on the eyes' initial fixation position in the words, and that on some occasions linguistic processing cancels the decisions made on the basis of these tactics. While this is compatible with the strategy-tactics theory (O'Regan, 1990,1992 ), our assumption is less extreme, since it considers within-word refixations to be influenced not only by predetermined oculomotor tactics but also by the efficiency of ongoing processing.

Our observation of a higher proportion of large saccades in the normal-text-reading control condition than in the three experimental conditions appears to be explicable in terms of differences in the skipping probability. Although the skipping rate was quite similar in the four scanning conditions, short words were skipped more often (in $5-10 \%$ of cases) in the normal-text-reading condition than in the three experimental conditions. This difference might be due to the fact that during normal reading, the upcoming word can sometimes be identified in parafoveal vision and does not, therefore, need to be fixated. As assumed in the strategy-tactics theory (O'Regan, 1990, 1992), the decision to skip a word would be made on the basis of predetermined oculomotor strategies, and only occasionally (e.g., when the duration of the prior fixation is long enough for the next word to be identified in parafoveal vision) would processing change a decision made on the basis of such strategies.

As mentioned above, the fact that the pattern of eye movements between and within words (or letter strings) is very similar-though not exactly the same-in the four conditions is consistent with a revised version of the strategy-tactics theory (O'Regan, 1990, 1992). During reading, the eyes would basically be driven by predetermined oculomotor strategy and tactics, with ongoing processing intervening occasionally, not only to modify the probability of skipping words or the fixation durations as assumed by O'Regan $(1990,1992)$, but also to modify within-word refixations. It is important to note, however, that an alternative (though less plausible) hypothesis might also account for the results obtained. According to such a hypothesis, during reading, eye movements would be mainly determined by the ongoing linguistic processing of the encountered words. In particular scanning situations (e.g., when the text is very easy to process or when less processing is required, as in the $z$-text-reading condition of the present experiment), the system would switch to an automatic oculomotor scanning mode based on the subject's reading experience. However, this all or none hypothesis seems rather unlikely, for if eye movements were determined in some situations by a processing-dependent scanning mode and in other situations by automatic oculomotor scanning, the refixation-probability and skipping-rate differences between the normal-text-reading condition and the three experimental conditions should not vary as a function of the encountered word lengths, as was observed in the present experiment.

Finally, as reported above, we have observed that fixation durations are globally shorter in the normal-textreading condition than in the three experimental conditions. The difference between normal-text reading and normal-text search probably results from the fact that in the search condition, word identification occurs prior to detection of the target letter, making fixation durations longer. This is compatible with the observation that fixation durations are, again, shorter in the normal-text-search condition than they are in the $z$-text-search condition, since although word identification would occur prior to letter detection, it would facilitate the search for the target letter. Indeed, it has been shown that a letter is more 
quickly detected in a word than it is in a meaningless letter string (Krueger, 1970).

On the other hand, the finding that fixation durations are shorter in the normal-text-reading condition than in the $z$-text-reading condition is opposite to that expected, since the scanning of meaningless letter strings requires less processing than does the reading of words (letter strings do not require lexical, syntactic, or semantic processing). The difference might, in fact, have resulted from the nature of the task given to the subjects; since in the $z$-text-reading condition the subjects were asked to move their eyes as though they were reading, they might have tried to keep their eyes still as long as possible at each fixation, overestimating the time they spend at each fixation during normal-text reading. Indeed, in another study, we observed longer fixation durations during normal-text reading than in a $z$-text-scanning condition, but in that study, the subjects had been asked to move their eyes as fast as possible from one letter string to another (Inhoff et al., 1993).

To summarize, we have shown that when the amount of linguistic (lexical, syntactic, and semantic) processing to be done is reduced, subjects are able to generate eyemovement patterns similar to those observed during normal-text reading. This suggests that predetermined oculomotor scanning strategies are an important element in determining eye movements in normal reading; in most instances, they might determine which word to go to next and how many fixations should be made on any given word. In particular instances, however, the foveal and/or parafoveal processing of the words that occurs in parallel with eye movements could affect the pattern of betweenand within-word eye movements. On the other hand, the time the eyes spend at each fixation in the text might be mainly determined by ongoing processing demands.

It is important to note that although it might be autonomous oculomotor scanning strategies that are basically driving the eyes during normal reading, such strategies might have been acquired during the process of learning to read, and might be a result of the effects of linguistic constraints on eye movements in the early stages of learning to read. One good example of such a constraint can be found in the strategy-tactics theory's (O'Regan, $1990,1992)$ explanation of the fact that within-word refixations are less frequent when the eyes are initially located near the middle of a word (the optimal viewing position effect). According to this explanation, such an effect results not directly from the fact that the processing of a word is more efficient from its middle (Nazir, O'Regan, \& Jacobs, 1991), but rather from predetermined oculomotor tactics based on the subject's experience that a word is more easily recognized from its middle.

\section{REFERENCES}

Balota, D. A., Pollatsek, A., \& Rayner, K. (1985). The interaction of contextual constraints and parafoveal visual information in reading. Cognitive Psychology, 17, 364-390.

Blanchard, H. E., Pollatsek, A., \& Rayner, K. (1989). The acqui- sition of parafoveal word information in reading. Perception \& Psychophysics, 46, 85-94

BOUma, H., \& DE VoOgd, A. H. (1974). On the control of eye saccades in reading. Vision Research, 14, 273-284.

BRILHL, D., \& INHOFF, A. W. (1995). Integrating information across fixations in reading: The use of orthographic bodies and of exterior letters. Journal of Experimental Psychology: Learning, Memory, \& Cognition, 21, 1-13.

BuswelL, G. T. (1920). An experimental study of the eye-voice span in reading. Supplementary Educational Monographs, 17.

CARPenter, P. A., \& Just, M. A. (1983). What your eyes do while your mind is reading. In K. Rayner (Ed.), Eye movements in reading: Perceptual and language processes (pp. 275-307). New York: Academic Press.

CoEfFÉ, C., \& O'Regan, J. K. (1987). Reducing the influence of nontarget stimuli on saccade accuracy: Predictability and latency effects. Vision Research, 27, 227-240.

DEARBORN, W. F. (1906). The psychology of reading. Archives of Philosophy, Psychology \& Scientific Methods, 4, 7-132.

EHRLICH, S. F., \& RAYNER, K. (1981). Contextual effects on word perception and eye movements during reading. Journal of Verbal Learning \& Verbal Behavior, 20, 641-655.

FindLAY, J. M. (1982). Global visual processing for saccadic eye movements. Vision Research, 22, 1033-1045.

FrAzIER, L., \& RAYNER, K. (1982). Making and correcting errors during sentence comprehension: Eye movements in the analysis of structurally ambiguous sentences. Cognitive Psychology, 14, 178210.

HABER, R. N. (1976). Control of eye movements in reading. In R. A. Monty \& J. W. Senders (Eds.), Eye movements and psychological processes (pp. 443-451). Hillsdale, NJ: Erlbaum.

Henderson, J. M., \& Ferreira, F. (1990). Effects of foveal processing difficulty on the perceptual span in reading: Implications for attention and eye movement control. Journal of Experimental Psychology: Learning, Memory, \& Cognition, 16, 417-429.

HochBeRg, J. (1975). On the control of eye saccades in reading. Vision Research, 15, 620 .

Hogaboam, T. W. (1983). Reading pattern in eye movement data. In $\mathrm{K}$. Rayner (Ed.), Eye movements in reading: Perceptual and language processes (pp. 309-332). New York: Academic Press.

HUEY, E. B. (1908). The psychology and pedagogy of reading. New York: Macmillan.

INHOFF, A. W. (1984). Two stages of word processing during eye fixations in the reading of prose. Journal of Verbal Learning \& Verbal Behavior, 23, 612-624

INHOFF, A. W. (1989a). Lexical access during eye fixations in reading: Are word access codes used to integrate lexical information across interword fixations? Journal of Memory \& Language, 28, 444461

INHOFF, A. W. (1989b). Parafoveal processing of words and saccade computation during eye fixations in reading. Journal of Experimental Psychology: Human Perception \& Performance, 15, 544-555.

INHOFF, A. W., \& RAYNER, K. (1986). Parafoveal word processing during eye fixations in reading: Effects of word frequency. Perception \& Psychophysics, 40, 431-439.

InhoFf, A. W., Topolski, R., Vitu, F., \& O'Regan, J. K. (1993). Attention demands during reading and the occurrence of brief (express) fixations. Perception \& Psychophysics, 54, 814-823.

JuOla, J. F., WARD, N., \& MCNAmaRA, T. (1982). Visual search and reading of rapid, serial presentation of letter strings, words and text. Journal of Experimental Psychology: General, 111, 208-227.

Just, M. A., \& CARPENTER, P. A. (1980). A theory of reading: From eye fixations to comprehension. Psychological Review, 87, 329-354.

JUST, M., \& CARPENTER, P. A. (1987). The psychology of reading and language comprehension. Newtown, MA: Allyn and Bacon.

KerR, P. W., \& McConkiE, G. W. (1992, July). Toward a mathematical description of eye movements during reading. Paper presented at the 25th International Congress of Psychology, Brussels.

Kolers, P. A. (1976). Buswell's discoveries. In R. A. Monty \& J. W. Senders (Eds.), Eye movements and psychological processes (pp. 371-395). Hillsdale, NJ: Erlbaum. 
KRUEGER, L. E. (1970). Search time in a redundant visual display. Journal of Experimental Psychology, 83, 391-399.

Lima, S. D., \& INhoff, A. W. (1985). Lexical access during eye fixations in reading: Effects of word-initial letter sequence. Journal of Experimental Psychology: Human Perception \& Performance, 11, 272-285.

McConkIE, G. W. (1979). On the role and control of eye movements in reading. In P. A. Kolers, M. E. Wrolstad, \& H. Bouma (Eds.), Processing of visible language (Vol. 1, pp. 37-48). New York: Plenum.

MCConkie, G. W., KerR, P. W., ReddiX, M. D., \& Zola, D. (1988). Eye movement control during reading: I. The location of initial eye fixations on words. Vision Research, 28, 1107-1118.

McConkie, G. W., Kerr, P. W., Reddix, M. D., Zola, D., \& Jacobs, A. M. (1989). Eye movement control during reading: II. Frequency of refixating a word. Perception \& Psychophysics, 46, 245-253.

MCCONKIE, G. W., Underwood, N. R., ZOLA, D., \& Wolverton, G. S. (1985). Some temporal characteristics of processing during reading. Journal of Experimental Psychology: Human Perception \& Performance, 11, 168-186.

Morrison, R. E. (1984). Manipulation of stimulus onset delay in reading: Evidence for parallel programming of saccades. Journal of Experimental Psychology: Human Perception \& Performance, 10, 667682.

NAZIR, T. A. (1991). On the role of refixations in letter strings: The influence of oculomotor factors. Perception \& Psychophysics, 49, 373-389.

NAZIR, T. A., O'Regan, J. K., \& JACOBS, A. M. (1991). On words and their letters. Bulletin of the Psychonomic Society, 29, 171-174.

O'REGAN, [J.] K. (1979). Saccade size control in reading: Evidence for the linguistic control hypothesis. Perception \& Psychophysics, 25, 501-509.

O'REGAN, J. K. (1990). Eye movements and reading. In E. Kowler (Ed.), Eye movements and their role in visual and cognitive processes (pp. 395-453). Amsterdam: Elsevier.

O'Regan, J. K. (1992). Optimal viewing position in words and the strategy-tactics theory of eye movements in reading. In $\mathrm{K}$. Rayner (Ed.), Eye movements and visual cognition: Scene perception and reading (pp. 333-354). New York: Springer-Verlag.

O'REGAN, J. K., \& LÉVY-SCHOEN, A. (1987). Eye movement strategy and tactics in word recognition and reading. In M. Coltheart (Ed.), Attention and performance XII: The psychology of reading (pp. 363383). Hillsdale, NJ: Erlbaum.

Pollatsek, A., Rayner, K., \& Balota, D. A. (1986). Inferences about eye movement control from the perceptual span in reading. Perception \& Psychophysics, 40, 123-130.
Pynte, J., Kennedy, A., \& Murray, W. S. (J991). Within-word inspection strategies in continuous reading: Time course of perceptual, lexical, and contextual processes. Journal of Experimental Psychology: Human Perception \& Performance, 17, 458-470

RAYNER, K. (1979). Eye guidance in reading: Fixation location within words. Perception, 8, 21-30.

RAYNER, K., \& DUFF, S. A. (1986). Lexical complexity and fixation times in reading: Effects of word frequency, verb complexity, and lexical ambiguity. Memory \& Cognition, 14, 191-201

RAYNER, K., \& MCCONKIE, G. W. (1976). What guides a reader's eye movements? Vision Research, 16, 829-837.

RAYNER, K., \& MORRIS, R. K. (1992). Eye movement control in reading: Evidence against semantic preprocessing. Journal of Experimental Psychology: Human Perception \& Performance, 18, 163-172.

RAYNER, K., \& POLlatsek, S. (1987). Eye movements in reading: A tutorial review. In M. Coltheart (Ed.), Attention and Performance XII: The psychology of reading (pp. 327-362). Hillsdale, NJ: Erlbaum.

RAYNER, K., \& POLIATSEK, S. (1989). The psychology of reading. London: Prentice-Hall.

SHEBILSKE, W. (1975). Reading eye movements from an informationprocessing point of view. In D. Massaro (Ed.), Understanding language (pp. 291-311). New York: Academic Press.

VITU, F. (1990). Le guidage oculaire pendant la lecture. Unpublished doctoral dissertation, University of Paris V.

VITU, F. (1991a). The existence of a center of gravity effect during reading. Vision Research, 31, 1289-1313.

VITU, F. (1991b). The influence of parafoveal preprocessing and linguistic context on the optimal landing position effect. Perception \& Psychophysics, 50, 58-75.

VITU, F. (1993). The influence of the reading rhythm on the optimal landing position effect. In G. d'Y dewalle \& J. Van Rensbergen (Eds.) Perception and cognition: Advances in eye movement research (pp. 181-192). Amsterdam: North-Holland.

Vitu, F., O'Regan, J. K., \& Mittau, M. (1990). Optimal landing position in reading isolated words and continuous text. Perception \& Psychophysics, 47, 583-600.

Viviani, P., \& SwensSon, R. G. (1982). Saccadic eye movements to peripherally discriminated visual targets. Journal of Experimental Psychology: Human Perception \& Performance, 8, 113-126.

ZOLA, D. (1984). Redundancy and word perception during reading. Perception \& Psychophysics, 36, 277-284.

(Manuscript received August 4, 1993; revision accepted for publication October 18,1994 .) 